\title{
In guter Gesellschaft
}

\section{Sylke Schröder}

Die "EthikBank « ist eine Direktbank für ethische und ökologische Geldanlagen, die »nachhaltiges Wirtschaften " auf ihre Fahnen geschrieben hat. Umgesetzt wird dies vor allem durch feste Anlagekriterien und mittels Positiv- und Negativlisten von Wirtschaftsunternehmen.

Der Betriebswirt versteht unter Nachhaltigkeit den wirtschaftlichen Umgang mit Vermögenswerten. Auf dem Deutschen Baugewerkstag 2000 äußerte sich Bundeskanzler Gerhard Schröder zur Nachhaltigkeit mit den Worten: »Wir dürfen nicht aufessen, worauf unsere Kinder und Kindeskinder angewiesen sind.«Dieses Zitat verweist auf einen Unterschied zum üblichen Sprachgebrauch und meint: Erzeuger und Nutznießer der Nachhaltigkeit sind nicht identisch.

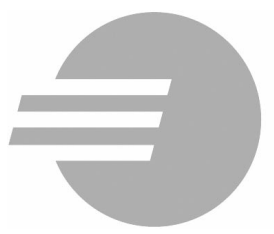

\section{EthikBank \\ Zweigniederlassung der Volksbank Eisenberg eG}

Der neue Inhalt des alten Begriffs entstand angesichts der Gefährdung unserer natürlichen Lebensgrundlagen. Die »Agenda 21 «machte ihn zum Leitbild. Und seitdem wurde er auf unzähligen Konferenzen, in dicken Umweltberichten, im Geschwall von Politikern und an runden Tischen von Nichtregierungsorganisationen zu dem gemacht, was er heute ist - eine geplättete Briefmarke.

Vor diesem Hintergrund ist jedes Unternehmen gut beraten, den Begriff mit Leben zu füllen und ihn aus der Abstraktion herauszuholen. Dieser Artikel beschreibt, was die EthikBank dafür tut.

\section{Ethische Orientierung gehört ins Kerngeschäft}

Ursprünglich auf den wirtschaftlichen Umgang mit Vermögenswerten beschränkt, verstehen wir heute unter Nachhaltigkeit den Dreiklang von Ökologie, Sozialem und Ökonomie. Viele Unternehmen meinen damit Spenden und Sponsoring. Es spricht nichts gegen ein gut strukturiertes Spendenkonzept, solange die Wohltaten nicht nach dem Gießkannenprinzip verteilt werden. Doch reicht das nach unserer Meinung nicht aus. Das Institut für ökologische Wirtschaftsforschung in Berlin fordert, ethische Orientierungen im Kerngeschäft umzusetzen. Das heißt, Ethik soll sich im Produkt selbst oder in den betrieblichen Abläufen widerspiegeln. Diesen Anspruch setzte die EthikBank mit ihrer Anlagepolitik um.

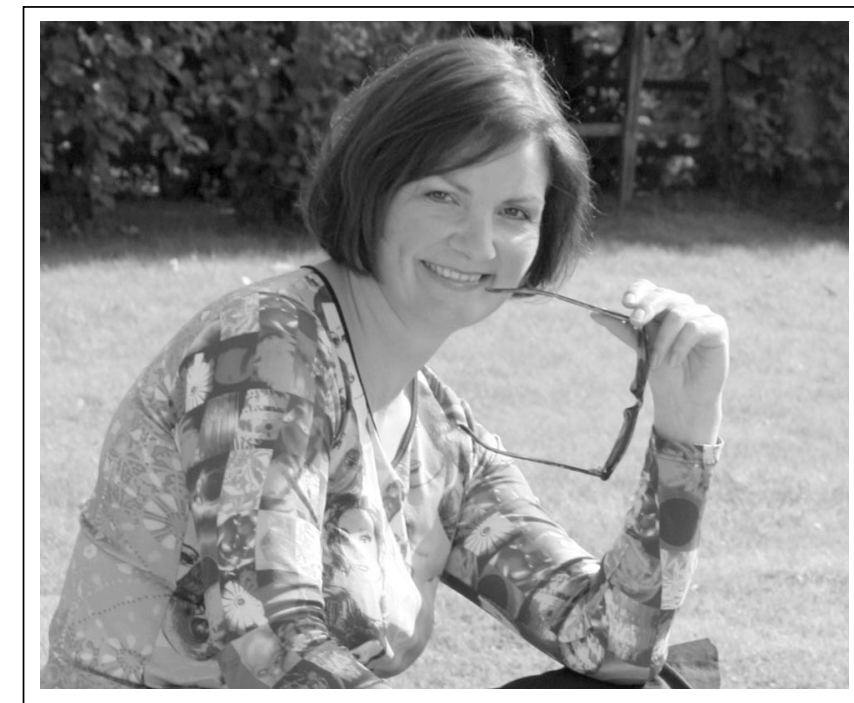

Sylke Schröder ist Prokuristin der EthikBank, eine Tochtergesellschaft der Volksbank Eisenberg. In der genossenschaftlichen Direktbank für ethisch-ökologische Geldanlage ist sie zuständig für Marketing, Vertrieb, Kommunikation und Qualitätsmanagement. Sylke Schröder wurde 1966 in Jena geboren und ist in Ostthüringen aufgewachsen. Nach einer Ausbildung als Bürokauffrau arbeitete sie als Sekretärin im Büro des Generaldirektors im Kombinat VEB Keramische Werke Hermsdorf und bildet sich zur »staatlich geprüften Sekretärin « weiter. Nach der Geburt ihres Sohnes 1987 nimmt sie ein Jahr später eine Stelle als Sekretärin in einer Töpferei an. In der Volksbank Eisenberg beginnt sie 1992 als Vorstandssekretärin, steigt zur Direktionsassistentin auf, kümmert sich um den Aufbau der Marketing- und Vertriebsorganisation und erhält schließlich im Jahr 2000 Prokura. Inzwischen ist sie ausgebildete Bankkauffrau, genossenschaftliche Bankbetriebswirtin, Marketingleiterin ADG und Vertriebsleiterin ADG.

E-Mail sylke.schroeder@ethikbank.de

\section{Die Anlagekriterien der EthikBank}

Die Anlagekriterien sind die Leitlinien der EthikBank. Dahinter steht die Idee, das Geld unserer Kunden für Mensch und Natur einzusetzen. Grundsätzlich hat eine Bank zwei Möglichkeiten, das Geld ihrer Kunden zu verwenden: Sie kann Kredite vergeben oder Wertpapiere am Kapitalmarkt kaufen. Die EthikBank ist nicht im Kreditgeschäft tätig; sie investiert das Geld unter ethischen Ge- 
sichtspunkten am Kapitalmarkt und hat dafür ein dreistufiges Filtersystem entwickelt:

- Erster Filter: Hier ist das Anlageuniversum fixiert - die Frage also, in welchen Segmenten des Kapitalmarktes die EthikBank agieren will. Das sind die Unternehmen des DAX und MDAX sowie Staaten.

- Zweiter Filter: Jetzt greifen die Tabukriterien. Das sind Zustände und Aktivitäten, die von der EthikBank abgelehnt werden. Bei Unternehmen gehören unter anderem Rüstung, Atomkraft und grüne Gentechnik dazu. Auch Kinderarbeit wird thematisiert. Politisch distanziert sich die Bank von solchen Staaten, die keine zivilen Rechte und demokratischen Grundfreiheiten gewähren, kurz: unfreie Staaten. Unternehmen und Staaten, die die Tabukriterien berühren, bleiben in diesem Filter hängen.

- Dritter Filter: Die verbleibenden Kandidaten werden auf Herz und Nieren untersucht, ehe sie den Sprung in die Positivliste der EthikBank schaffen. Die wichtigsten Posi-

ber. Wir sind davon überzeugt, dass Nachhaltigkeit mit gutem Willen in jedem Unternehmen möglich ist. Der Bewerber wird in den Bewerbungsunterlagen auf Bereiche gestoßen, die in seinem Unternehmen noch brachliegen. Dadurch soll er animiert werden, die Nachhaltigkeit weiter zu entwickeln und sich nicht auf den Lorbeeren des Erreichten auszuruhen. Auch solche Unternehmen, die sich bisher noch nicht um eine nachhaltige Wirtschaftsweise bemühen, sollen zum Denken angeregt werden.

Deshalb sucht der Preis sowohl Ökobetriebe als auch konventionelle Branchen. Ob ein Unternehmen aus innerer Überzeugung nachhaltig wirtschaftet oder aus pragmatischen Gründen, ist für uns nicht relevant. Wichtig ist, die Unternehmer der Tat zu finden - solche, die nicht nur reden, sondern handeln. Der Förderpreis wurde bisher einmal vergeben. Das war vor einem Jahr. Die öffentliche Resonanz war enorm. Mit Alnatura, ein Handelsunternehmen für Bio-Produkte in Hessen, und der Soli fer Solardach $\mathrm{GmbH}$ in Sachsen gingen der erste und zweite Preis an Ökobetriebe. Darunter verstehen wir solche Unternehmen, die ein umweltfreundliches Produkt herstellen. Dass auch konventionelle Unternehmen respektable soziale und ökologische Leistungen erreichen können, bewies die Hering Bau GmbH \& Co.KG aus Burbach in Nordrhein-Westfalen mit dem dritten Platz.

\section{und Ökonomie«}

tivkriterien sind Arbeitsplatzerhalt, Umgang mit Kunden, Lieferanten und Mitarbeitern, Gleichberechtigung, Menschenrechte und Leistungen im Umweltbereich. Außerdem profitieren solche Unternehmen, die präventive Maßnahmen zur Korruptionsvorsorge getroffen haben. Im Ergebnis dieser letzten Untersuchung bleiben jene Unternehmen übrig, die in der Summe der genannten Positivkriterien als Branchenbeste abschneiden; ebenso die überdurchschnittlich guten OECD-Staaten.

Die Positivliste der Unternehmen wird aktuell von der Firma Henkel angeführt. Auf der Staatenliste haben die skandinavischen Länder sowie die deutschsprachigen Nachbarn die Nase vorn. Die EthikBank veröffentlicht ihre Positivlisten im Internet. Aber sie geht noch einen Schritt weiter. Im Internet sind auch die eigenen Geldanlagen abgebildet. So können Kunden ihrer EthikBank jederzeit auf die Finger schauen und selbst prüfen, ob wir uns an unsere heiligen Anlagegrundsätze halten.

\section{Der Förderpreis »Nachhaltiger Mittelstand»}

Die EthikBank stellt hohe Anforderungen an die ethische Qualität der Staaten und Unternehmen, in die sie das Geld ihrer Kunden investiert. Da aber im DAX und MDAX naturgemäß nur Konzerne vertreten sind, gibt es ein weiteres Instrument: ein Förderkonzept für Mittelständler. Die Förderung des Mittelstandes gehört zu den erklärten Zielen der EthikBank. Neben mittelstandsfreundlichen Produkten mit und ohne Fördercharakter ist die EthikBank Stifterin des Förderpreises »Nachhaltiger Mittelstand «.

Dieser Förderpreises würdigt die Leistungen der Preisträger. Aber in erster Linie verstehen wir ihn als Impulsge-
Ursprünglich wollten wir den Förderpreis jährlich ausschreiben - bis wir gemerkt haben, wie viel Arbeit dahinter steckt. Deshalb wird der Preis künftig alle zwei Jahre vergeben. Die Ausschreibung des 2. Förderpreises begann im November 2005.

\section{Die Förderprojeke}

Das Herz der EthikBank sind die Anlagekriterien und das Förderkonzept. Der Förderpreis ist ein Element dieses Förderkonzeptes. Aber es gibt drei weitere Förderprojekte, die die EthikBank langfristig unterstützt: ein Frauen-, ein Ethik- und ein Umweltprojekt. Dahinter stehen eine Mädchenschule in Afghanistan, ein Waisenhaus in Bulgarien und die bedrohte Baikalrobbe in Sibirien. Diese Projekte unterstützen wir langfristig durch eigene Spenden und durch unsere Öffentlichkeitsarbeit. Wir schütten unsere Spenden also nicht mit der Gießkanne aus. Wir haben uns bewusst entschieden, unsere Zeit und unsere finanziellen Mittel nur in diese Projekte zu investieren. Die Transparenz unserer Projektpartner ist uns besonders wichtig, weil wir unseren Kunden gegenüber für die Qualität und die Glaubwürdigkeit unserer Partner verbürgen.

Auch unsere Kunden können diese Projekte unterstützen. Wer sich für ein Förderkonto entscheidet, verzichtet auf einen Teil seiner Zinsen und spendet diese dem Projekt seiner Wahl. Darüber hinaus organisieren wir regelmäßig Direktspenden-Aktionen, die von unseren Kunden gut angenommen werden.

Dabei haben wir eine interessante Erfahrung gemacht. Viele Kunden berichten uns, dass sie gern für unsere Projekte spenden, weil sie genau wissen, wofür ihr Geld verwendet wird. Denn die EthikBank informiert ihre Kunden regelmäßig über Fortschritte und den aktuellen Projekt- 
stand. Das macht keine der großen Spendenorganisationen.

\section{Das »MikroKonto« - das Konto für Schuldner}

Im Mai letzten Jahres hat die EthikBank das »MikroKonto« auf den Markt gebracht. Damit hat sich erstmals in der Geschichte Deutschlands eine Bank für eine Zielgruppe entschieden, die von anderen Banken gemieden wird: Es handelt sich um ein Guthabenkonto für überschuldete Menschen. Wer erst einmal in die Schuldenfalle getappt ist, weiß, was es heißt, ohne Girokonto leben zu müssen. Die Betroffenen haben ohne Konto keine Chance, eine Arbeit oder eine Wohnung zu bekommen. Der Weg ins soziale Aus ist vorprogrammiert. Die Nachfrage nach dem »MikroKonto « ist groß. Wir fragen diese Menschen nicht nach den Gründen ihrer Überschuldung. Wir wollen nur sehen, dass sie ihr Problem aktiv lösen. Das "MikroKonto« eröffnen wir für alle Menschen, die ihre Schulden gerichtlich oder außergerichtlich reguliert haben.

\section{Würde die EthikBank ihren eigenen Förderpreis gewinnen?}

In der EthikBank dreht sich alles um Anlagekriterien, Förderprojekte und Förderprodukte. Damit hat die Bank ihre ethischen Grundwerte ins Kerngeschäft geholt. Man sollte meinen, schon deshalb wäre die EthikBank auch selbst eine Kandidatin für ihren eigenen Förderpreis natürlich nur als Gedankenspiel.

Hinzu kommt, dass sie sich an ihren Positivkriterien messen lässt. Entgegen dem Branchentrend baute die EthikBank ihre Arbeitsplätze deutlich aus. Im Dezember 2002 startete sie mit zwei Mitarbeitern. Heute beschäftigt die Bank sechs Mitarbeiter. 50 Prozent der Führungskräfte sind Frauen. Viel Geld investiert sie in Personalentwicklung und Weiterbildung. Jeder Mitarbeiter hat ein Recht auf ein jährliches Beurteilungsgespräch. Die Beteiligung der Mitarbeiter am Jahresgewinn, Geldleistungen zu persönlichen Anlässen, die von der Bank finanzierte private Zusatz-Krankenversicherung, Betriebs- und Weihnachstsfeiern oder kostenfreie Getränke - all das sind nicht die großen Errungenschaften. Aber in der Summe machen diese das Leben in einem leistungsorientierten Unternehmen wie der EthikBank angenehm.

Die Stärken der EthikBank liegen im sozialen Bereich. In Umweltfragen haben wir Nachholebedarf. Die konsequente Verwendung von Umweltpapier und der Einsatz von einseitig bedrucktem Papier für interne Zwecke ist bisher das Einzige, was wir uns in Sachen Umwelt auf die Fahnen schreiben können. Aber wir entwickeln immer weiter und haben Spaß daran.

\section{Die Tabukriterien der EthikBank}

Die Bank investiert nicht in Unternehmen, die

- Militärwaffen herstellen oder vertreiben

- Atomkraftwerke besitzen oder betreiben

- Pflanzen und Saatgut gentechnisch verändern

- ozonzerstörende Chemikalien herstellen oder vertreiben

- Kinderarbeit zulassen

\section{Die fünf Besten der Positivliste}

- Henkel

- Degussa

- Allianz

- BMW

- Merck

\section{Fünf Unternehmen der Negativliste}

- Telekom (Rüstung)

- Siemens (Rüstung)

- Volkswagen (Rüstung)

- E.on (Atomkraft)

- BASF (grüne Gentechnik)

\section{Förderpreis »Nachhaltiger Mittelstand»}

Der Preis zeichnet mittelständische Unternehmen aus, die in ihrer täglichen Arbeit Ökonomie, Ökologie und Soziales langfristig in Einklang bringen. Nachhaltigkeit ist in jedem Unternehmen möglich, deshalb sucht der Förderpreis sowohl »richtige « Ökobetriebe als auch konventionelle Unternehmen. Die Ausschreibung des Förderpreises begann im November 2005 und endet im April 2006.

\section{Förderprojekte und Förderprodukte}

- Mädchenschule in Khancharbagh (Afghanistan)

- Waisenhaus »Königin Maria Luisa« in Plovdiv (Bulgarien)

- bedrohte Baikalrobbe (Sibirien)

Im letzten Jahr förderte die EthikBank diese drei Projekte mit insgesamt 15.000 Euro. Aus Kundenspenden kamen mehr als 9.500 Euro zusammen. Jeder Kunde kann bei der Förderung mitmachen. Es gibt zwei Möglichkeiten: die Entscheidung für ein Förderkonto oder die Beteiligung an einer Direktspenden-Aktion.

EthikBank, Zweigniederlassung der Volksbank Eisenberg eG, Martin-Luther-Straße 2, 07607 Eisenberg, Telefon 036691 862345, Fax 036691 58555, E-Mail sylke.schroeder@ethikbank.de, Internet http://www.ethikbank.de 